\title{
Representing a democratic constituency in negotiations: Delegation vs. Ratification
}

\author{
Daniel Cardona · Clara Ponsatí
}

March, 2015

\begin{abstract}
We consider negotiations where one of the parties is a group that must send a representative to the bargaining table. We examine the trade-offs that the group faces in choosing between two different regimes for this representation: (i) Delegation where the representative is granted full authority to reach an agreement, and (ii) Ratification, where any agreement reached by the representative requires a posterior ratification vote. We show that when the group has flexibility - to select the delegate or to set the majority threshold for ratification - the majority of the group favors delegation. Only when the flexibility is limited or delegates are (sufficiently) unreliable will the majority of the group prefer ratification.
\end{abstract}

Keywords: Delegated bargaining; ratification requirement; fast-track authority; majority voting. JEL Classification: C7, F5

\section{Introduction}

Consider a group - composed of individuals with common but not identical interests - that wishes to set up a procedure for their joint negotiations with external parties. The group is large, so they must be represented at the bargaining table. Will the majority of the group prefer to appoint a delegate with full authority, or would they rather submit the agreements reached by their representative to a ratification vote? Who will be their delegate, or how strong a majority will they require for ratification?

In this paper we examine these questions in the context of a simple model where a continuum of individuals, the group, must agree on how to share some surplus with a third party. All individuals want as large a share as possible for the group, but their valuations in case of disagreement are heterogeneous. We assume that the representation regime for negotiation is decided by a simple majority vote, and that this collective choice may include the selection of the delegate and/or a (super)

We acknowledge financial support from the Generalitat de Catalunya through grant SGR2009-1051, and from the Ministerio de Economía y Competitividad through grants ECO2009-08820 and ECO2012-34046.

Daniel Cardona (Corresponding author): Universitat de les Illes Balears and CREB. Departament d'Economia Aplicada, Campus UIB, 07122 Palma de Mallorca, Illes Balears, Spain. Ph: +43-971172845. Fax: +43-971172389. e-mail: d.cardona@uib.cat.

Clara Ponsatí: University of St. Andrews, Institut d'Anàlisi Econòmica - CSIC and Barcelona GSE. e-mail: clara.ponsati@st-andrews.ac.uk 
majority threshold for ratification. Representation procedures are decided at a stage where the details of the negotiations - in particular, what surplus is available - are uncertain. We show that, when the group has flexibility, so that their choices include every (super)majority threshold for ratification, and delegates with the full range of mandates (reservation values), then the majority of the group favors delegation over ratification. It is only when this flexibility is limited, or when delegates are sufficiently unreliable, that the majority of the group may prefer a regime of ratification. Furthermore, we show that the selected delegate is always more extremist (less inclined to agreement) than the median voter. Hence, our results imply that democratic constituencies have a tendency to provide themselves with representation procedures for negotiation that (with positive probability) deliver disagreements that the median voter regrets ex-post. Interestingly, when delegates are unreliable, the preferences of the median member of the group over the bargaining regime and those of the opponent are not necessarily in conflict.

The game-theoretical literature addressing negotiations of groups vis a vis a common opponent is scarce. ${ }^{1}$ The basic model that we borrow for our analysis is due to Haller and Holden (1997). They examine the role of ratification requirements for collective negotiations; they point out that ratification votes act as a commitment device that increases bargaining power à la Schelling (1960), and they conclude that groups have a tendency to self-impose super-majority ratification requirements for their collective negotiations. Manzini and Mariotti (2005) propose a model where, at each stage of the bargaining game, it is the whole group - not a representative - that, following some internal procedure, formulates and accepts/rejects proposals. They characterize the unique equilibrium and display how the group internal procedures, whether majoritarian or unanimous, affect the bargaining outcome.

Our results also contribute to the literature on legislative organizations and procedures. First, we contribute to the theory of negotiations in bicameral systems. Gailmard and Hammond (2011) examine the creation of committees that are delegated to carry out negotiations with the other chamber. They assume an ultimatum game bargaining procedure, where one chamber makes a take it or leave it offer to the other. They find that endowing the committee with both gatekeeping and agenda setting power leads to (optimally) electing unrepresentative committees, i.e. committees whose median member is more extremist than the chambers median. Our results can be directly interpreted in this context, with the advantage that our model does not rely on assuming ultimatum power for one of the two chambers. Another interpretations of our results contributes to the discussion on presidential "fast-track" authority for international negotiations. This literature (see for example Tarar, 2006 or Conconi et al., 2012 ) examines the legislatures dilemma between granting the president "fast-track authority" or keeping amendment power over agreements. Under "fast-track" authority, the legislature can only ratify proposals transmitted by the president, which has a direct interpretation as our ratification regime. In contrast, a regime where the legislature keeps amendment power is the analogous to our delegation regime. We discuss this interpretation and its implications in more detail in section 6 .

As our main focus of interest is the collective nature of the negotiation and the democratic election of procedures within the group, we have abstracted from informational asymmetry considerations, that have been a main concern of the wealth of literature addressing the costs and benefits of delegation. ${ }^{2}$ Naturally, informational issues might also be relevant in our context, in which case the benefits of delegation that we have uncovered ought to be weighted with additional agency costs and benefits. Another major concern in delegation might be that the possibility of renegotiation undermines its

\footnotetext{
1 Most papers analyzing strategic delegation in bargaining environments consider that either a single player or a constituency with all members having the same preferences, selects a representative. As a small sample, see Jones (1989), Burtraw (1992), Segendorff (1998), Cai (2000), Cai and Cont (2004), Harstad (2008), Chae (2009) or Christiansen (2013).

2 See Aghion and Tirole (1997) and Dessein (2002).
} 
positive effects. However, in our context the delegate is an elected member of the group, who subsequently enters the negotiation with her own reservation value. Furthermore, as argued by Bester and Sákovics (2001), in bargaining games, commitment through delegation retains its value even under the possibility of renegotiation.

The rest of the paper is organized as follows. Section 2 describes the model. Section 3 examines the choice of procedures in situations where the identity (reservation value) of the delegate and the threshold for ratification are set. In section 4 we examine the richer set ups where the group can elect the delegate or set what (super) majority is necessary for ratification, and chooses between the two. Section 5 addresses situations where the reliability of delegates is limited. Section 6 interprets our model in the context of the "fast-track" authority discussion. Section 7 concludes.

\section{The model}

The basic set up is that of Haller and Holden (1997). Two parties, must bargain on how to share a divisible surplus $s$. One of the parties is a large group $N$, whereas the other party, the opponent, is a single individual that obtains 0 in case of disagreement. An agreement splits the surplus allocating a share $a \in[0, s]$ to the group and the remainder $s-a$ to the opponent. All the members of the group get utility $a$ upon agreement, but in case of disagreement they each get reservation utility $x_{i} \in[0,1]$, $i \in N$, which vary across individuals. The cumulative distribution of reservation utilities, denoted by $F$, has a positive density $f$ over $(0,1)$. We write $m$ to denote the median individual of the group; that is, $F\left(x_{m}\right)=1 / 2$.

The group bargains through a representative, and this can take place in two different bargaining modes. The first regime are negotiations by delegation, where the representative is granted full authority to reach any agreement. We refer to her as the delegate, and denote her reservation value as $x_{d}$. The second regime are negotiations subject to ratification. In this case, the representative must submit her agreement with the opponent to a ratification vote. We refer to her as the spokeswoman, and denote her reservation value as $x_{s w} \cdot{ }^{3}$ An agreement $a$ must be ratified by the approval of a (super)majority $q \in[1 / 2,1]$. Thus, $a$ is approved provided that it is not smaller than the reservation utility of the pivotal voter $x_{q}$, where $F\left(x_{q}\right)=q$. We will refer to a bargaining regime to a bargaining mode with its associated either delegate or ratification requirement.

A fundamental feature of the setup is that the individuals of the group are ex-ante uncertain about the amount of (gross) surplus to share with the opponent. This surplus is revealed only after negotiations with the opponent start. The realized surplus is a drawn from a random variable $s \sim[0,1]$ with cumulative distribution $G$, which is independent of $F$. For convenience we assume that $G$ has a positive density $g$ over $(0,1)$, and that both are continuously differentiable.

\subsection{The bargaining outcome}

Once the representative meets the opponent to bargain, all the data of the bargaining problem, the bargaining regime, the available surplus $s$, the reservation value of the representative $x$, and the the ratification threshold $q$, if relevant, are complete information to both parties. For simplicity we take a reduced form approach to model this bilateral bargaining situation: If the bilateral net surplus $s-x$ is positive the two parties agree to split it in equal shares, paying attention to the ratification

\footnotetext{
3 The preferences of the agents are not affected by the identity of the representative. This differs from Cai (2000) where, in a different setting, the representative gets utility from her role.
} 
constraint only if it is binding. ${ }^{4}$ If the ratification constraint is binding, then the group gets $x_{q}$, the minimal share that passes the ratification vote. If the net surplus is negative, disagreement prevails. Thus, the bargaining outcomes are as follows:

1. Under delegation there is agreement at $a_{D}=\left(x_{d}+s\right) / 2$ provided that $x_{d} \leq s$; and otherwise disagreement prevails.

2. Under ratification, an agreement $a_{R}$ will be approved only if $a_{R} \geq x_{q}$. Thus, the agreed share will be $a_{R}=\max \left\{\left(s+x_{s w}\right) / 2, x_{q}\right\}$ whenever $s \geq x_{q}$ and $s \geq x_{s w}$; otherwise disagreement prevails.

Observe that, if $s$ was known prior to selecting the negotiation regime this selection is trivial. In particular, with flexibility to select the identity of the delegate or the ratification requirement, the group could extract all the surplus from the opponent in either regime: A delegate with $x_{d}=s$ would obtain all the surplus for the group. Likewise, the same outcome would prevail in negotiations subject to ratification with an spokeswoman with reservation value $x_{s w}=0$ and a ratification threshold $q=F(s) .^{5}$ Thus, our assumption that bargaining regime is set at a stage where $s$ is still uncertain is what makes the problem interesting.

It is immediate that sending spokeswoman with $x_{s w}=0$ subject to a ratification threshold $q$ or delegating to the individual with reservation value $x_{d}=x_{q}$ do not yield equal bargaining outcomes. The reason is simple: In delegated negotiations, $x_{d}$ is effectively the reservation value of the group, which affects the bargaining outcome for all $s$. On the other hand, under ratification $x_{q}$ plays the role of an outside option - and it affects the agreed share only when it is binding.

Congruently, taking $\left(x_{d}, x_{s w}, q\right)$ as given, the ex-ante expected utilities of individual $i$ for each regime are as follows:

1. Under delegation, with $x=x_{d}$

$$
U_{i}^{D}(x)=G(x) x_{i}+\int_{x}^{1}((z+x) / 2) d G(z)
$$

where the first term refers to the situation when no agreement is reached, and so $i$ gets $x_{i}$, and the second term is the expected payoff conditional on an agreement being reached.

2. Under ratification, with $x=x_{q}$

$$
U_{i}^{R}\left(x_{s w}, x\right)=G\left(\max \left\{x_{s w}, x\right\}\right) x_{i}+\int_{\max \left\{x_{s w}, x\right\}}^{1} \max \left\{\frac{z+x_{s w}}{2}, x\right\} d G(z)
$$

where we express the dependence of expected utilities on $q$ indirectly via the reservation utility of the pivotal voter $x_{q}$.

Although with different nuances, these expected utilities highlight the trade-off between selecting a though representative versus one who is likely to obtain agreement: A large value of $x$ increases the bargaining outcome when there is agreement at the expense of reducing the probability of such agreement.

\footnotetext{
4 This is the outcome in the Nash solution, which prevails as the limit of equilibria under the usual non-cooperative alternating proposals formulations.

5 A different matter is whether this is sufficient for the group: If $F(s) \leq 1 / 2$ (that is, the reservation utility of the median voter's is higher than $s$ ), a majority would prefer a disagreement.
} 


\subsection{The majority choice}

Due to the group heterogeneity, their members have different preferences over bargaining regimes. Hence, to assess group decisions, we will need to examine the individual preferences of the group members over the range of feasible alternatives. These preferences will usually take the form of a utility $u_{i}$ over alternatives $x \in[0,1]$. The following properties are useful to discuss collective choices:

Single-Peakedness We say that individual utility $u_{i}$ over $[0,1]$ is single-peaked (SP) if there is a unique alternative $\hat{x} \in[0,1]$ with $u_{i}(\hat{x}) \geq u_{i}(x)$ for all $x \in[0,1]$ such that $u_{i}\left(x^{\prime}\right)>u_{i}(x)$ for any $0<x<x^{\prime}<\hat{x}$, and $u_{i}\left(x^{\prime}\right)<u_{i}(x)$ for any $\hat{x}<x<x^{\prime}<1$.

Single-Crossing We say that individual utilities $u_{i}$ and $u_{j}$ over $[0,1]$ satisfy single-crossing (SC) if for $i<j$ and $x<x^{\prime}$ the following holds: i) $u_{j}(x)>u_{j}\left(x^{\prime}\right) \Rightarrow u_{i}(x)>u_{i}\left(x^{\prime}\right)$, and ii) $u_{i}\left(x^{\prime}\right)>$ $u_{i}(x) \Rightarrow u_{j}\left(x^{\prime}\right)>u_{j}(x)$.

Majority Preference We say that alternative $x$ is majority-preferred to alternative $x^{\prime}$, if there is a simple majority of players preferring $x$ to $x^{\prime}$.

When the majority preference over a choice set is transitive, then there is a unique (set of) alternative(s) which is stable in the sense that no other alternative is superior for all members of a simple-majority coalition. These alternatives (which may exist even when the majority preference is not transitive), are known as Condorcet winners. It is well known that the existence of Condorcet winners is guaranteed when the utilities of any two individuals in a group are are single-peaked and/or satisfy single-crossing. These results are referred in the literature as the the Median Voter Theorem (Black, 1958), and the Representative Voter Theorem (Rothstein, 1991), respectively. When they exist, Condorcet winners are a robust prediction of the group's decision, particularly "for situations in which people can act in concert, with various subsets of people coordinating their actions to form coalitions....for unilaterally insuring an improvement in the welfare of all of its members." (Ordeshook, 1980). Hence, we will assume that decisions within the group involve procedures selecting a Condorcet winner, when it exists. ${ }^{6}$

\section{Choosing the negotiation regime without flexibility.}

First, we address the comparison between delegation and ratification in situations where both the identity of the representative and the ratification threshold are fixed. That is, the group faces a binary choice between delegation with $x_{d}$ or ratification with $\left(x_{s w}, x_{q}\right) \cdot{ }^{7}$ It is immediate that, since only two alternatives are available, the majority preference is transitive so that there is a Condorcet winner. Moreover, independently of the identity of the representatives and the ratification requirement, the majority choice coincides with the most preferred alternative of the median, as shown next. ${ }^{8}$

Proposition 1 Given $\left(x_{d}, x_{s w}, x_{q}\right)$, the median's preferred alternative is the Condorcet winner.

\footnotetext{
6 Group decisions may strongly depend on the exact procedure used by the group, and consequently some decision rules may select outcomes different from the Condorcet winner - as in bureaucratic models with monopoly on the agenda à la Niskanen (1971). In a standard alternating offer majority bargaining game, Banks and Duggan (2000) display an example with a two-dimension policy space and single-peaked preferences such that non-Concorcet winners may be attained as equilibrium outcomes.

7 One may think, for instance, in scenarios where a legislature decides to grant full authority to the President or require that any agreement must be ratified by some qualified majority in the Congress. In these cases, the spokeswoman and the delegate coincide.

8 It is worth to anticipate the importance of this result on our further analysis: It allows to characterize majority decisions between any two different mode bargaining regimes.
} 
Proof Let first assume that $x_{q}>x_{s w}$, and define

$$
T(i)=U_{i}^{D}\left(x_{d}\right)-U_{i}^{R}\left(x_{s w}, x_{q}\right) .
$$

We prove that $T(i) \geq 0$ implies that either (i) $T(j) \geq 0$ for all $j$ with $x_{i}>x_{j}$ or (ii) $T(j) \geq 0$ for all $j$ with $x_{i}<x_{j}$. A similar argument applies when $T(i) \leq 0$. Let

$$
G\left(x_{d}\right) x_{i}+\int_{x_{d}}^{1} \frac{z+x_{d}}{2} d G(z) \geq G\left(x_{q}\right) x_{i}+\int_{x_{q}}^{1} \max \left\{\frac{z+x_{s w}}{2}, x_{q}\right\} d G(z),
$$

and assume $T(j)<0$ for some $j$ with $x_{i}>x_{j}$. That is,

$$
G\left(x_{d}\right) x_{j}+\int_{x_{d}}^{1} \frac{z+x_{d}}{2} d G(z)<G\left(x_{q}\right) x_{j}+\int_{x_{q}}^{1} \max \left\{\frac{z+x_{s w}}{2}, x_{q}\right\} d G(z) .
$$

Adding the inequalities we obtain

$$
G\left(x_{d}\right)\left[x_{i}-x_{j}\right]>G\left(x_{q}\right)\left[x_{i}-x_{j}\right] .
$$

Thus, if (i) is not satisfied we obtain $x_{d}>x_{q}$. A similar argument proves that if $T(j)<0$ for some $j$ with $x_{i}<x_{j}$ then we conclude that $x_{d}<x_{q}$. Hence, we obtain a contradiction and the claim follows. In case that $x_{q} \leq x_{s w}, U_{i}^{R}\left(x_{s w}, x_{q}\right)=U_{i}^{D}\left(x_{s w}\right)$ and a similar reasoning applies.

The optimal bargaining regime for the median voter - and thus the group's choice - can be either of the two. As an illustration, the next example shows that, given $x_{d}$ and $x_{s w}$, the median may prefer delegation to the ratification regime for both small and high values of $x_{q}$, while ratification is preferred for intermediate values of $x_{q}$.

Example 1 Assume $G(z)=z^{2}, g(z)=2 z, x_{s w}=x_{d}=0.32$ and $x_{m}=0.3$. Using Eqs. (1) and (2), we obtain $U_{m}^{D}\left(x_{d}\right)=0.49675$ and

$$
U_{m}^{R}\left(x_{s w}, x_{q}\right)=\left\{\begin{array}{ll}
0.49675 & \text { if } x_{q} \leq 0.32 \\
0.33 x_{q}^{3}-0.34 x_{q}^{2}+0.1 x_{q}+0.49 & \text { if } x_{q} \in[0.32,0.66] \\
x_{q}\left(1-x_{q}^{2}+0.3 x_{q}\right) & \text { if } x_{q}>0.66
\end{array} .\right.
$$

These functions are depicted in Figure 1.

The distribution of surplus $G(z)$ in the example is standard (log-concave), yet it does not guarantee the single-peakedness of $U_{m}^{R}\left(x_{s w}, x_{q}\right)$ with respect to $x_{q}$. This implies that there is no clear cut-off determining the preference of the median (and thus of the majority) over the bargaining regimes. Nevertheless, there are specifications of the model that induce a single-peak utility function $U_{m}^{R}$, in which case the preference of the majority is easily determined. For instance, single-peakedness is assured when the surplus is uniformly distributed and $x_{s w}=x_{d}=x$ (we omit the proof, which follows directly from Eq. (2)). ${ }^{9}$

Proposition 2 Given $x_{d}=x_{s w}$ and $s \sim U[0,1]$ then either delegation is preferred to ratification for all $x_{q}$ or there exists a threshold $x_{\bar{q}}$ such that the ratification is preferred iff $x_{q} \leq x_{\bar{q}}$.

9 Haller and Holden (1997) considered a representative with no explicit minimal requirements so that $x_{s w}=0$. In this environment, they showed that $g^{\prime}(s)>0$ assures the single-peakedness of $U_{i}^{R}\left(0, x_{q}\right)$. Thus, the next proposition can be also extended to these cases. 


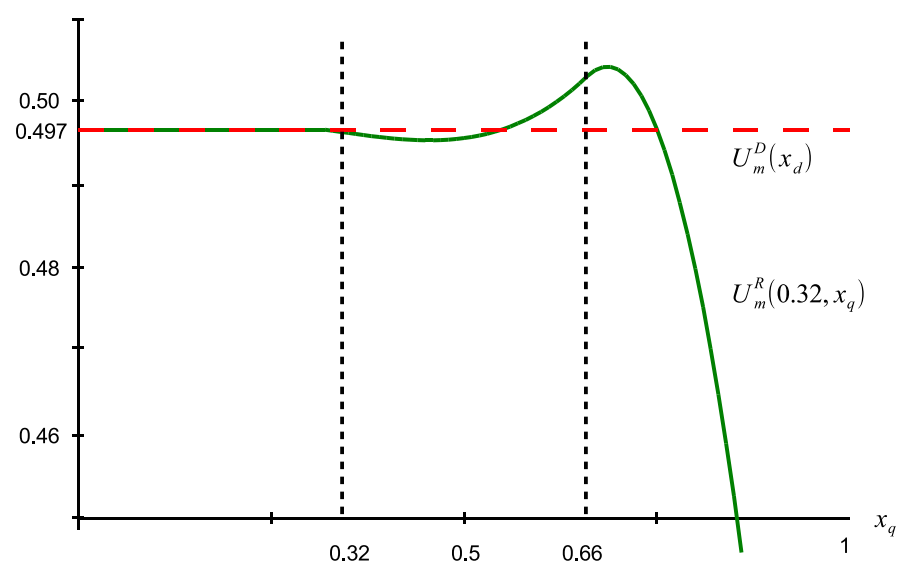

Fig. 1: Non single-peakedness of $U_{m}^{R}\left(x_{s w}, x_{q}\right)$ with respect to $x_{q}$

When the identities of representatives are fixed and equal, the ratification requirement has two effects: First, it precludes any agreement when the surplus is small $\left(s<x_{q}\right)$; and second, it increases (at least weakly) the surplus obtained by the legislature when the realization $s$ is greater than the minimal share required by the pivotal agent $x_{q}$. While the second effect has a positive impact on the median's expected utility, the first one may be negative when the reservation value of the median is smaller than that of the pivotal player. Then, the preference over the bargaining mode would depend on how these effects counterbalance.

\section{Choosing the negotiation regime with flexibility}

We have seen that the preference of the group over the bargaining mode when both the reservation utility of the representatives and the ratification requirement are fixed depends on the exact values of these parameters. Nevertheless, for any bargaining regime the agents have some preferences about the identity of the representatives and/or the ratification requirement. Thus, when the group has flexibility to select them it is not necessarily true that any bargaining mode might be selected by the group. In particular, we next show that delegation prevails.

Let us now assume that, in addition to selecting the negotiation mode, the group has flexibility to elect the delegate - that is, her reservation value $x_{d}$ - or the ratification threshold $x_{q}$, while the reservation value of the spokeswoman is fixed at $x_{s w} \in\left\{0, x_{m}\right\} .{ }^{10}$ For any $i$, we denote by $x_{i}^{D}$ and $x_{i}^{R}$ the values that maximize $U_{i}^{D}(x)$ and $U_{i}^{R}\left(x_{s w}, x\right)$, respectively.

Next, we show that when the bargaining mode is given (either delegation or ratification) then the preferences of the agents satisfy SC so that the Representative Voter theorem applies to the alternatives within each regime.

10 Both, Haller and Holden, 1997 and Perry and Samuelson, 1994 consider that the reservation of the spokeswoman is zero. We also consider the possibility that she is the median. 
Lemma 1 Assume that the bargaining mode is fixed. Then, the preferences of the agents satisfy SC.

Proof Assume first the the bargaining mode is delegation, and consider $i, j$ with $x_{i}<x_{j}$ and $x_{d}<x_{d}^{\prime}$. SC requires that: (i) $U_{j}^{D}\left(x_{d}\right)>U_{j}^{D}\left(x_{d}^{\prime}\right) \Rightarrow U_{i}^{D}\left(x_{d}\right)>U_{i}^{D}\left(x_{d}^{\prime}\right)$, and (ii) $U_{i}^{D}\left(x_{d}^{\prime}\right)>U_{i}^{D}\left(x_{d}^{\prime}\right) \Rightarrow$ $U_{j}^{D}\left(x_{d}^{\prime}\right)>U_{j}^{D}\left(x_{d}\right)$. Suppose, by contradiction, that $U_{j}^{D}\left(x_{d}\right)>U_{j}^{D}\left(x_{d}^{\prime}\right)$ and $U_{i}^{D}\left(x_{d}\right) \leq U_{i}^{D}\left(x_{d}^{\prime}\right)$. Then,

$$
G\left(x_{d}\right) x_{j}+\int_{x_{d}}^{1}\left(\left(z+x_{d}\right) / 2\right) d G(z)>G\left(x_{d}^{\prime}\right) x_{j}+\int_{x_{d}^{\prime}}^{1}\left(\left(z+x_{d}^{\prime}\right) / 2\right) d G(z)
$$

and

$$
G\left(x_{d}\right) x_{i}+\int_{x_{d}}^{1}\left(\left(z+x_{d}\right) / 2\right) d G(z) \leq G\left(x_{d}^{\prime}\right) x_{i}+\int_{x_{d}^{\prime}}^{1}\left(\left(z+x_{d}^{\prime}\right) / 2\right) d G(z)
$$

Subtracting these inequalities, we obtain

$$
G\left(x_{d}\right)\left[x_{j}-x_{i}\right]>G\left(x_{d}^{\prime}\right)\left[x_{j}-x_{i}\right]
$$

Since $x_{j}-x_{i}>0$ and $G\left(x_{d}\right)<G\left(x_{d}^{\prime}\right)$ this is a contradiction. This proves part (i). Part (ii) can be similarly proved. Consider now that the bargaining model is ratification, with $x_{s w}$ fixed, and let $i, j$ with $x_{i}<x_{j}$ and $x_{q}<x_{q}^{\prime}$. Suppose that $U_{j}^{R}\left(x_{s w}, x_{q}\right)>U_{j}^{R}\left(x_{s w}, x_{q}^{\prime}\right)$ and $U_{i}^{R}\left(x_{s w}, x_{q}\right) \leq U_{i}^{R}\left(x_{s w}, x_{q}^{\prime}\right)$; that is,

$$
\begin{aligned}
& G\left(\max \left\{x_{s w}, x_{q}\right\}\right) x_{j}+\int_{\max \left\{x_{s w}, x_{q}\right\}}^{1} \max \left\{\frac{z+x_{s w}}{2}, x_{q}\right\} d G(z) \\
& >G\left(\max \left\{x_{s w}, x_{q}^{\prime}\right\}\right) x_{j}+\int_{\max \left\{x_{s w}, x_{q}^{\prime}\right\}}^{1} \max \left\{\frac{z+x_{s w}}{2}, x_{q}^{\prime}\right\} d G(z)
\end{aligned}
$$

and

$$
\begin{aligned}
& G\left(\max \left\{x_{s w}, x_{q}\right\}\right) x_{i}+\int_{\max \left\{x_{s w}, x_{q}\right\}}^{1} \max \left\{\frac{z+x_{s w}}{2}, x_{q}\right\} d G(z) \\
& \leq G\left(\max \left\{x_{s w}, x_{q}^{\prime}\right\}\right) x_{i}+\int_{\max \left\{x_{s w}, x_{q}^{\prime}\right\}}^{1} \max \left\{\frac{z+x_{s w}}{2}, x_{q}^{\prime}\right\} d G(z) .
\end{aligned}
$$

Adding up the previous inequalities yields,

$$
G\left(\max \left\{x_{s w}, x_{q}\right\}\right)\left[x_{j}-x_{i}\right]>G\left(\max \left\{x_{s w}, x_{q}^{\prime}\right\}\right)\left[x_{j}-x_{i}\right],
$$

which is a contradiction. Symmetrically, assuming $U_{i}^{R}\left(x_{s w}, x_{q}^{\prime}\right)>U_{i}^{R}\left(x_{s w}, x_{q}\right)$ and $U_{j}^{R}\left(x_{s w}, x_{q}^{\prime}\right) \leq$ $U_{j}^{R}\left(x_{s w}, x_{q}\right)$ would imply

$$
G\left(\max \left\{x_{s w}, x_{q}^{\prime}\right\}\right)\left[x_{i}-x_{j}\right]>G\left(\max \left\{x_{s w}, x_{q}\right\}\right)\left[x_{i}-x_{j}\right]
$$

again a contradiction. Hence single-crossing holds.

By the Representative Voter Theorem, the previous lemma implies that, if a Condorcet winner exists then it must be either the median's optimal delegation regime or the median' optimal ratification regime. By Proposition 1, the majority choice between any specific delegation regime and any specific ratification requirement, follows the median's preference. Thus, when all the alternatives are available there is a Condorcet winner that coincides with the median' preferred negotiation regime, and this Condorcet winner is the median's optimal delegation regime. 
Proposition 3 The median's optimal delegation regime is the Condorcet winner.

Proof From Proposition 1 and Lemma 1, it is immediate that the majority choice must be the median's preferred regime. Moreover, Lemma 3 in the Appendix states that $x_{m}^{R}>x_{s w}$. From (1) and (2) it is immediate that

$$
U_{m}^{D}\left(x_{m}^{D}\right) \geq U_{m}^{D}\left(x_{m}^{R}\right) \geq U_{m}^{R}\left(x_{m}^{R}, x_{s w}\right)
$$

The previous result bears on the different effect that delegation generates in the negotiations. As commented in Section 2.1., $x_{d}$ affects monotonically the bargaining outcome when negotiations end up with agreement. However, a ratification requirement of $q$ affects negotiations only when agents anticipate that a share smaler that $x_{q}$ would not be ratified by the legislature. For this reason, delegating to a representative with disagreement $x_{d}$ is always preferred to selecting a ratification requirement $q$ satisfying $x_{q}=x_{d}$. Thus, when the legislature has flexibility to appoint either a delegate or the ratification requirement, the median would prefer even a suboptimal delegate over the best ratification threshold. ${ }^{11}$ This dominance of delegation as a commitment device delivers the remarkable result that the median's optimal delegation regime is the Condorcet winner even in case that committee decisions are made simultaneously over any possible bargaining regime. ${ }^{12}$

\section{Unreliable delegates}

We have so far stress the importance of delegation as a commitment instrument compared with the use of a ratification requirement. Nevertheless, giving full authority to the delegate may have some drawbacks because the legislature may lose control over the decisions taken by the delegate. This is not an problem under complete information about the identity of the delegate, as assumed previously, since the behavior of delegates can be fully anticipated. However, the potential benefits of delegation may be eroded when there is uncertainty about his reservation value, because an unreliable delegate may either (i) reach agreements in situations where the median would prefer disagreement or (ii) reach no agreement in situations where the available surplus is such that the median would agree. While the latter is also present in the complete information setting (in fact, this is the commitment aspect of delegation), the former introduces a new distortion, which is similar to the one previously discussed in Section 3.

In order to introduce the uncertainty that delegation might involve, let us next examine situations where only a signal of the reservation utility of each group member $x_{i}$ is publicly observed. Moreover, due to a change of status, once appointed as a delegate, the reservation utility of the representative may change. For simplicity, assume that the disagreement of the delegate $x_{d}$ follows a "Bernoulli" distribution: with probability $p$ the signal is reliable and the true reservation value is $x_{d}$, and with probability $1-p$ it is equal to $0 .{ }^{13}$ Thus, the expected utility of individual $i$ when a delegate with reliability $p$ and signal $x_{d}$ is appointed is

$$
V_{i}^{D}\left(x_{d}, p\right)=\left\{\begin{array}{l}
p U_{i}^{D}\left(x_{d}\right)+(1-p) U_{i}^{D}(0) \text { if } x_{d} \neq x_{i} \\
p U_{i}^{D}\left(x_{d}\right)+(1-p) U_{0}^{D}(0) \text { if } x_{d}=x_{i}
\end{array}\right.
$$

Note that

$$
U_{i}^{D}(0)=U_{0}^{D}(0)=\bar{U}=\int_{0}^{1} \frac{z}{2} d G(z),
$$

11 We thank a referee for this remark.

12 Note that SP and SC are properties of preferences defined on the one-dimensional policy space. That is, for any given bargaining mode.

13 By assuming that when the true reservation utility is unrelated to $x_{i}$ then it is drawn from $F$, things would not change. 
so that,

$$
V_{i}^{D}\left(x_{d}, p\right)=p U_{i}^{D}\left(x_{d}\right)+(1-p) \bar{U} .
$$

It is easy to check that these preferences satisfy SC and that the optimal delegate of agent $i$ coincides with her optimal choice under certainty, $x_{i}^{D}$. The difference with respect to the situation with certainty is that now, $V_{i}^{D}\left(x_{i}^{D}, p\right)$ may be smaller that $U_{i}^{R}\left(x_{s w}, x_{i}^{R}\right)$, which is not affected by uncertainty. Moreover, now the probability of agreement under delegation is enhanced due to the positive probability that the delegate would have a zero reservation utility.

Our next result compares the preferences of the median player between delegation and ratification under this type of uncertainty.

Lemma 2 There exists $\bar{p}_{m} \in[0,1]$ such that $m$ prefers delegation over ratification if and only if her partners are reliable with probability $p \geq \bar{p}_{m}$.

Proof Individual $m$ prefers delegation over ratification if and only if

$$
p U_{m}^{D}\left(x_{m}^{D}\right)+(1-p) \bar{U} \geq U_{m}^{R}\left(x_{s w}, x_{m}^{R}\right)
$$

where the left hand side of the inequality is increasing in $p$. Hence, since $U_{m}^{D}\left(x_{m}^{D}\right) \geq U_{m}^{R}\left(x_{s w}, x_{m}^{R}\right)$, then either the left hand side dominates for all $p$, or there or there exists $\widetilde{p}_{m} \in(0,1)$ satisfying

$$
\widetilde{p}_{m} U_{m}^{D}\left(x_{m}^{D}\right)+\left(1-\widetilde{p}_{m}\right) \bar{U}=U_{m}^{R}\left(x_{s w}, x_{m}^{R}\right)
$$

Thus, delegation is preferred to ratification for $p \geq \bar{p}_{m}=\max \left\{0, \widetilde{p}_{m}\right\}$.

The median player prefers agreements being subject to ratification only when the delegate is (sufficiently) unreliable. Note, however, that there may exists parameter configurations such that $\bar{p}_{m}=0$, implying that delegation might be preferred by the median player regardless of unreliability.

Our next result establishes that the negotiation regime selected by simple majority will ultimately depend on the probability threshold $\bar{p}_{m}$ of the median. That is, the median's optimal regime is the Condorcet winner.

Proposition 4 If delegates are reliable with probability $p$, the Condorcet winner is $x_{m}^{D}$ if $p \geq \bar{p}_{m}$, otherwise it is $x_{m}^{R}$.

Proof Using a similar reasoning as in Lemma 1, it can be checked that for any bargaining regime the preferences satisfy SC. Thus, the candidates to be the Condorcet winner are either $x_{m}^{D}$ or $x_{m}^{R}$. Moreover, Proposition 1 can also easily adapted to show that the median's best choice prevails.

Table 1 displays some numerical examples for $x_{s w}=0$ when $F$ and $G$ are beta distributions with parameters $\left(a_{1}, b_{1}\right)$ and $\left(a_{2}, b_{2}\right)$, respectively. Note that the reservation value of the median's optimal delegate $x_{m}^{D}$ is always lower than $x_{m}^{R}$, the reservation value of the pivotal voter under the median's optimal ratification regime. This indicates that the probability of agreement is greater under delegation. Note also that, although the expected utility of the median player decreases with uncertainty and the outcome under the ratification is not affected by uncertainty, when the precision on the delegate reservation value is high, delegation is still preferred to ratification. 


\begin{tabular}{lcccccccc}
$\left(a_{1}, b_{1}\right)$ & $\left(a_{2}, b_{2}\right)$ & $x_{m}$ & $x_{m}^{D}$ & $x_{m}^{R}$ & $U_{m}^{D}$ & $\bar{U}$ & $U_{m}^{R R}$ & $\bar{p}$ \\
\hline$(1,3)$ & $(1,3)$ & 0.206 & 0.319 & 0.398 & 0.268 & 0.125 & 0.248 & 0.86 \\
$(1,3)$ & $(1,1)$ & 0.206 & 0.470 & 0.603 & 0.415 & 0.25 & 0.363 & 0.685 \\
$(1,3)$ & $(3,1)$ & 0.206 & 0.588 & 0.685 & 0.605 & 0.375 & 0.531 & 0.678 \\
$(1,1)$ & $(1,3)$ & 0.500 & 0.571 & 0.625 & 0.510 & 0.125 & 0.507 & 0.992 \\
$(1,1)$ & $(1,1)$ & 0.500 & 0.667 & 0.750 & 0.583 & 0.25 & 0.562 & 0.937 \\
$(1,1)$ & $(3,1)$ & 0.500 & 0.710 & 0.782 & 0.685 & 0.375 & 0.647 & 0.877 \\
$(3,1)$ & $(1,3)$ & 0.793 & 0.822 & 0.844 & 0.793 & 0.125 & 0.792 & 0.998 \\
$(3,1)$ & $(1,1)$ & 0.793 & 0.862 & 0.869 & 0.806 & 0.25 & 0.804 & 0.996 \\
$(3,1)$ & $(3,1)$ & 0.793 & 0.868 & 0.900 & 0.829 & 0.375 & 0.822 & 0.985 \\
$(1,10)$ & $(1,1)$ & 0.066 & 0.377 & 0.533 & 0.356 & 0.25 & 0.284 & 0.321 \\
\hline
\end{tabular}

Table 1

\section{6 "Fast-track" authority vs. amendment power}

Our results admit an interpretation that provides insight on the trade-offs that legislatures face when deciding between granting the executive "fast-track" authority or keeping amendment powers. A legislature granting "fast-track" authority to the executive, can only approve or reject a negotiated deal, but cannot amend it. That is, a regime of "fast-track" authority is the equivalent to our ratification regime. On the other hand, when a legislature maintains amendment powers, then legislators are involved in negotiations with the opponent. Conconi at al. (2012) assume that, with amendment power, the legislature can always make a final take-it-or-leave-it proposal. Hence, in their model amendment power implies that the legislature extracts all the surplus and leaves the opponent at her reservation value. Not surprisingly, they conclude that legislatures always prefer to keep amendment power, but potential trade partners are disinclined to begin negotiations. In their setup, "fast-track" authority is favored only as a mean to promote participation by the opponent. But the legislature direct involvement in bargaining, does not necessarily imply full surplus extraction. If the opponent has symmetric bargaining power, then the amendment power regime is precisely as delegated bargaining in our formulation. In this interpretation, our results imply that: First, "fast-track" authority (ratification) is the inferior regime if the legislature has great flexibility and can set freely both the reservation value for amendments and the majority threshold for ratification. Second, when the reservation value for amendments is fixed (say at the reservation value of the median voter in the trade committee of the legislature) and ratification demands a given (super)majority, then both regimes can prevail as the top choice for a majority of the legislature. Lastly, when there is uncertainty on the reservation value that will be relevant for amendments, "fast-track" may be the preferred regime.

Although not conclusive, the simulations in Table 1 suggest that, under flexibility and perfect reliability, the probability of agreement is higher under delegation $\left(x_{m}^{D}<x_{m}^{R}\right)$, and therefore the preferences of the opponent over the negotiation regime need not be opposed to those of the group median. The conflict of interest over procedures with negotiation counterparts was stressed in Conconi et al. (2012). Our results provide a more nuanced insight on this issue, as we display conditions under which both sides agree that delegation is the best procedure for negotiations. In fact, in our model the conflict of interest over procedures remains important, provided that the reservation value of the legislature can be reliably determined, because the extra surplus delivered by delegation will be fully appropriated by the group. Nevertheless, when delegates are unreliable things change. Uncertainty has two effects: (i) it reduces the expected payoff of the median, and simultaneously (ii) increases the probability of agreement. Therefore, it is possible that the group and the opponent agree that delegation is the best regime.

We can explicitly display natural parametrizations for which the group and the opponent agree on what regime is best. Lemma 3 in the Appendix establishes that that $x_{m}^{R} \geq x_{s w}$. Therefore, the 
expected utility of the opponent in each bargaining regime is given by

$$
\begin{aligned}
V_{o}^{D}\left(x_{m}^{D}, p\right) & =p \int_{x_{m}^{D}}^{1}\left(\frac{z-x_{m}^{R}}{2}\right) d G(z)+(1-p) \bar{U} \\
U_{o}^{R}\left(x_{s w}, x_{m}^{R}\right) & =\int_{x_{m}^{R}}^{1} \min \left\{\frac{z-x_{s w}}{2}, z-x_{m}^{R}\right\} d G(z) .
\end{aligned}
$$

Now, consider the values displayed in the Table 1 under parameters $\left(a_{1}, b_{1}\right)=\left(a_{2}, b_{2}\right)=(1,3)$. Note that when $p=0.95$ we obtain that $V_{m}^{D}\left(x_{m}^{D}, p\right)>U_{m}^{R}\left(x_{s w}, x_{m}^{R}\right)$. Moreover, evaluating $V_{o}^{D}\left(x_{m}^{D}, p\right)$ and $U_{o}^{R}\left(x_{s w}, x_{m}^{R}\right)$ at $x_{m}^{D}=0.319, x_{m}^{R}=0.398$ and $p=0.95$ we obtain $V_{o}^{D}\left(x_{m}^{D}\right)=0.03179<$ $0.032618=V_{o}^{R}\left(x_{s w}, x_{m}^{R}\right)$. Likewise, when $p=0.9$ we still have that $V_{m}^{D}\left(x_{m}^{D}, p\right)>U_{m}^{R}\left(x_{s w}, x_{m}^{R}\right)$. However, now $V_{o}^{D}\left(x_{m}^{D}, p\right)=0.036696>0.032618=U_{o}^{R}\left(x_{s w}, x_{m}^{R}\right)$. That is, when delegation is preferred by the group and the reliability of the delegate is relatively small, there are no conflicting interests between the group and the opponent with respect to the bargaining regimes, and $x_{m}^{D}$ is preferred to $\left(x_{s w}, x_{m}^{R}\right)$ by the two parties.

\section{Conclusions}

In this paper, we analyzed the choice made by a group of individuals when selecting procedures that will govern their negotiations with third parties. Specifically, we considered situations where one of the members of the group must be selected to represent them in the negotiations. We studied the trade-offs that the group faces when choosing between two alternative negotiation modes: either to select a delegate with full authority to reach any agreement or to use a ratification procedure to confirm any agreement reached by the representative. We have shown that when the identity of the representative is common knowledge and simple majority is required to set the bargaining regime, then delegation is always selected. Only when delegates are (sufficiently) unreliable will the majority of the group prefer ratification.

We have also contributed to the literature addressing legislatures deciding between granting a delegate "fast-track" authority or keeping its amendment power. In our model, the choice faced by the committee is richer, as it consists on choosing either a delegate with full authority or a representative whose agreement is subject to a ratification vote of the legislature. However, the basic insights of the two approaches are similar: When delegates are reliable, a majority of agents in the constituency prefers delegation. This means that the legislature - implicitly, through the endogenously selected delegate - can make amendments (or counter-offers) in the negotiations, which contrast to "fasttrack" negotiations where the role of the legislature is limited to a ratification vote. Additionally, we showed that the preferences of the opponent over the bargaining mode are not necessarily negatively correlated preferences with the median's preferences when delegates are unreliable. The reason is that alternative bargaining modes generate different probabilities of agreement - therefore, a different available surplus - and that the unreliability of the delegate may favor the position of the opponent.

\section{Appendix}

Lemma $3 x_{m}^{R} \geq x_{m}$ for all $x_{m}<1$. 
Proof Haller and Holden (1997) proved the result when $x_{s w}=0$. We next prove that this is also the case when $x_{s w}=x_{m}$. Let consider the optimal ratification requirement of $m$. That is, $x_{m}^{R}$ maximizes

$$
U_{m}^{R}\left(x_{s w}, x\right)=\left\{\begin{array}{lr}
G\left(x_{s w}\right) x_{m}+\int_{x_{s w}}^{1} \frac{z+x_{s w}}{2} d G(z) & \text { if } x \leq x_{s w} \\
G(x) x_{m}+\int_{x}^{\min \left\{2 x-x_{s w}, 1\right\}} x d G(z)+\int_{\min \left\{2 x-x_{s w}, 1\right\}}^{1} \frac{z+x_{s w}}{2} d G & \text { if } x \geq x_{s w}
\end{array} .\right.
$$

Clearly, $U_{m}^{R}\left(x_{s w}, x\right)$ is constant if $x \leq x_{s w}$. Moreover, the function is continuous at $x=x_{s w}$, and for $x \geq x_{s w}$ the right derivative satisfies

$$
\frac{\partial U_{m}^{R}\left(x_{s w}, x\right)}{\partial x}>g(x) x_{m}-g(x) x \geq 0 \text { at } x=x_{m}<1 .
$$

Thus, $x_{m}^{R}>x_{m}$ for all $x_{m}<1$.

\section{References}

1. Aghion, P. and J. Tirole (1997) "Formal and Real Authority in Organizations" Journal of Political Economy, $105,1-29$.

2. Banks, J.S. and J. Duggan (2000) "A Bargaining Model of Collective Choice" American Political Science Review 94, 73-88.

3. Bester, H. and J. Sákovics (2001) "Delegated Bargaining and Renegotiation" Journal of Economic Behavior and Organization 45 (4), 459-473.

4. Black, D. (1958) "The Theory of Committees and Elections" Cambridge: Cambridge University Press.

5. Burtraw, D. (1992) "Strategic Delegation in Bargaining" Economics Letters 38, 181-185.

6. Cai, H. (2000) "Bargaining on Behalf of a Constituency" Journal of Economic Theory 92, 234-273.

7. Cai, H. and W. Cont (2004) "Agency Problems and Commitment in Delegated Bargaining" Journal of Economics and Management Strategy 13 (4), 703-729.

8. Chae, S. (2009) "Group Bargaining with Representation" Seoul Journal of Economics 22 (2), 245-262.

9. Conconi, P., G. Facchini and M. Zanardi (2012) "Fast Track Authority and International Trade Negotiations" American Economic Journal: Economic Policy 4 (3), 146-189.

10. Christiansen, N. (2013) "Strategic Delegation in a Legislative Bargaining Model with Pork and Public Goods" Journal of Public Economics 97, 217-229.

11. Dessein, W. (2002) "Authority and Communication in Organizations" Review of Economic Studies 69, 811-838.

12. Gailmard, S. and Hammond, T.(2011) "Intercamaral Bargaining and Intracameral Organization in Legislatures" The Journal of Politics 73 (2), 535-546.

13. Haller, H. and Holden, S. (1997) "Ratification Requirement and Bargaining Power" International Economic Review 38 (4), 825-851.

14. Harstad, B. (2008) "Do Side Payments Help? Collective Decisions and Strategic Delegation" Journal of the European Economic Association 6, 468-477.

15. Jones, S.R.G. (1989) "Have your Lawyer call my Lawyer. Bilateral Delegation in Bargaining Situations" Journal of Economic Behavior and Organization 11, 159-174.

16. Manzini, P. and Mariotti, M. (2005) "Alliances and Negotiations" Journal of Economic Theory 121, 128-141.

17. Niskanen, W. A. (1971) "Bureaucracy and representative government" Chicago: Aldine-Atherton.

18. Ordeshook, P.C. (1980) "Political Disequilibrium and Scientific Inquiry: A Comment on William Riker's "Implications from the Disequilibrium of Majority Rule for the Study of Institutions" "The American Political Science Review 74 (2), 447-450.

19. Perry, M. and Samuelson, L. (1994) "Open- versus Closed-door Negotiations" RAND Journal of Economics 25 (4), 348-359.

20. Rothstein P. (1991) "Representative Voter Theorems" Public Choice 72, 193-212.

21. Schelling, T.C. (1960) "The Strategy of Conflict" Harvard University Press, Cambridge, Massachusetts.

22. Segendorff, B. (1998) "Delegation and Threat in Bargaining" Games and Economic Behavior 23, 226-283.

23. Tarar, A. (2006) "Bargaining Power, the Schelling Conjecture, and "Fast-Track" Trade Negotiating Authority" Unpublished work. 